\title{
Polar localization of a dihydropyridine receptor on living Fucus zygotes
}

\author{
Sidney L. Shaw and Ralph S. Quatrano* \\ Department of Biology, University of North Carolina, Chapel Hill, NC 27599-3280, USA \\ ${ }^{*}$ Author for correspondence
}

\section{SUMMARY}

We have used a fluorescently-labeled dihydropyridine (FLDHP) to vitally stain living Fucus zygotes during the establishment of cell polarity. Localization of FL-DHP is primarily at the plasma membrane and FL-DHP binding is competitively blocked by an unlabeled dihydropyridine. Distribution of FL-DHP is initially symmetrical before fixation of the polar axis, but becomes asymmetrical in response to a unilateral light gradient. The distribution of FL-DHP receptors can be relocalized when the direction of the photopolarizing stimulus is changed. Treatment of cells with cytochalasin $B$ prior to axis fixation reversibly prevents localization of FL-DHP receptors. Observation of FL-DHP labeling by time-lapse fluorescence microscopy indicates that the existing receptors are redistributed during polar axis formation. The asymmetric distribution of FL-DHP receptors coincides temporally and spatially with increased local intracellular calcium ion concentrations, as measured by calcium green dextran. Based on the site, timing, photo-reversibility, and actin dependence of the asymmetric localization of FL-DHP receptors, we conclude that FL-DHP is a vital probe for the later stage of polar axis formation in Fucus zygotes. Furthermore, we propose that FL-DHP receptors correspond to ion channels that are transported to the future site of polar growth to create the changes in local calcium concentration required for polarity establishment.

Key words: Polarity, Fluorescent-dihydropyridine (FL-DHP), DMBODIPY, Calcium, Fucus, Embryogenesis

\section{INTRODUCTION}

Polar cell divisions occur throughout plant development and are critical for stomate development (Wick, 1991), trichome initiation (Hülskamp et al., 1994), gamete formation (McCormick, 1993; Reiser and Fischer, 1993), and the establishment of the developmental axis in the embryo (Jürgens et al., 1991; Jürgens, 1995). Very little is known about the mechanisms of polar cell division in plant cells and less so for plant zygotes, which are somewhat inaccessible to direct observation and manipulation. Zygotes of the intertidal Fucales, Pelvetia and Fucus, serve as an excellent model system to study the establishment of cell polarity (Goodner and Quatrano, 1993; Kropf, 1992, 1994; Fowler and Quatrano, 1995). The newly fertilized zygote is free of surrounding tissue and has a labile polar axis (Quatrano, 1978). An axis of polarity can be experimentally imposed upon the zygotes and continually reoriented during the first half of the zygotic cell cycle using unilateral white light, electric current, or with any of several other vectoral stimuli (Quatrano, 1973). The irreversible commitment to a polar axis (polar axis fixation; PAF) is assayed in zygotes as the point in time when the polar axis can no longer be reoriented by changing the direction of the incident vector (Quatrano, 1973).

Several factors are required for PAF in Fucale zygotes. It has been demonstrated (Quatrano, 1973) that Fucus zygotes do not fix an axis in the presence of cytochalasin, indicating a requirement for an intact F-actin cytoskeleton. Furthermore, a localized concentration of actin is found at the site of polar outgrowth at the time of PAF (Brawley and Robinson, 1985; Kropf et al., 1989). In addition to the actin cytoskeleton, an intact cell wall is required for PAF (Kropf et al., 1988). Fucus protoplasts incubated in a mixture of enzymes that hydrolyze cell wall components are developmentally competent but fail to fix a polar axis.

Far less is known about how the polar axis is initially formed prior to PAF. A localized ion influx, composed partially of calcium ions, appears before the polar axis is fixed and marks the site of future polar growth (Jaffe, 1966; Robinson and Jaffe, 1975; Nuccitelli, 1978). An ion sensitive vibrating probe has been used to show that the localized ion influx, and an accompanying localization of jelly secretion, are relocated to another region of the cell by changing the direction of incident light (Nuccitelli, 1978). The ability to change the position of ion influx and jelly secretion indicates that these localization events are correlated with the period corresponding to a labile polar axis, i.e. polar axis formation. Furthermore, it has been shown by Quatrano (1973) that the formation of the polar axis is cytochalasin sensitive, and later by Brawley and Robinson (1985), that disruption of the actin cytoskeleton with cytochalasin D prevented the majority of localized ion influx. All of these studies suggest that formation of a polar axis involves an actin dependent localization of ion channels, some of which are calcium channels (Quatrano et al., 1979; Brawley and Robinson, 1985; Kropf and Quatrano, 1987; Quatrano, 1990; Fowler and Quatrano, 1995). Although there is still much 
speculation about the role of calcium ions in the establishment of polarity, localized calcium ion influx is one of the earliest observable polar phenomena in zygotes and suggests that calcium channels might serve as molecular markers for observing the formation of the polar axis.

In this report, we employ a fluorescent-dihydropyridine (FLDHP) probe, originally designed for the labeling and purification of mammalian calcium channels (Knaus et al., 1992), on living Fucus zygotes to determine if FL-DHP receptors would reveal asymmetries that arise during the establishment of cell polarity. Our data indicate that the FL-DHP binds to a specific plasma membrane localized receptor that becomes polarly distributed prior to the fixation of the polar axis. Also, that the dihydropyridine receptor is associated with regions of elevated calcium concentration in the cell cortex. The asymmetric localization of the probe is actin dependent and can be redistributed by reversing the orientation of incident unilateral light. On the basis of these observations, we conclude that the FL-DHP is a vital marker for the later stage of polar axis formation in Fucus, and discuss a possible mechanism for early polarity establishment based on the actin dependent translocation of plasma membrane bound ion channels.

\section{MATERIALS AND METHODS}

Source material (Fucus disticus (L.) Powell) was collected from Yaquina Head and shipped from Newport, OR and stored at $4^{\circ} \mathrm{C}$ for up to 3 weeks. Synchronous populations of fertilized zygotes were obtained by rinsing ripe receptacles for 10 minutes in $4^{\circ} \mathrm{C}$ tap water followed by submersion in artificial sea water (ASW) at $16^{\circ} \mathrm{C}$ for 30 minutes. Zygotes were collected by filtering through a $90 \mu \mathrm{m}$ nylon screen and 3 successive rinses in ASW. Cells were allowed to develop in darkness, or placed in unidirectional fluorescent light (1,000 lux or $3,000 \operatorname{lux}$ ) for 1 to 12 hours, at $16^{\circ} \mathrm{C}$. Assays for the determination of polar axis fixation were performed by plating cells in unilateral light at 3 hours after fertilization (AF) and $180^{\circ}$ reversal of one slide each hour from 6 to 14 hours AF. The percentage of cells with a fixed polar axis was determined by counting the number of cells at 24 hours with rhizoids oriented to the first light direction versus the second light direction.

Vital staining of Fucus zygotes was performed by incubating cells in ASW at a final concentration of $1.6 \mu \mathrm{M}$ to $5 \mu \mathrm{M}(4,4$-difluoro-5,7dimethyl-4-boro-3a,4adiaza)-3-(s-indacene)propionic acid (-) DMBODIPY-DHP (green) or (4,4-difluoro-7-steryl-4-bora-3a,4a-diaza)3-(s-indacene)propionic acid (-) ST-BODIPY-DHP (red) (Molecular Probes, Eugene, Oregon) diluted from a $500 \mu \mathrm{M}$ ethanol stock, for no less than 5 minutes and for as long as 48 hours. Activated charcoal was used in some cases to remove unbound FL-DHP. ( \pm ) BAY-K 8644 was purchased from Calbiochem, San Diego, CA. Free BODIPY dye was obtained from Molecular Probes (Eugene, Oregon) as a succinimidyl ester and converted to a carboxyl by incubation in aqueous solution at $\mathrm{pH} 9.0$ for 24 hours. Assays for the light induced polar localization of the fluorescent dihydropyridine (FL-DHP), either the green DM-BODIPY-DHP or the red ST-BODIPY-DHP were performed by scoring all cells for asymmetric staining, that were not within $80 \mu \mathrm{m}$ of another zygote, for localized fluorescence.

DM-BODIPY-DHP was observed using a Zeiss inverted microscope, a $10 \times$ Plan, $16 \times$ Plan or $40 \times$ Neofluor objective lens, and an epifluorescence filter set containing a $550 \pm 20 \mathrm{~nm}$ emission filter. Images were collected using a CCD camera modified for on-chip integration and controlled using NIH-Image 1.55 software (written by Wayne Rasband at the US National Institutes of Health and available from the internet by anonymous FTP from zippy.nimh.nih.gov or on floppy disk from the National Technical Information Service, Springfield, Virginia, part number PB93-504868) as fully described by Shaw et al. (1995). Time-lapse digital fluorescence microscopy was performed using a computer controlled shuttering device for both epifluorescence and side-on (light vector) illumination. Confocal microscopy was performed with a Zeiss inverted CLSM 410 using a $40 \times$ oil immersion 1.4 NA objective lens. Laser illumination at 488 $\mathrm{nm}$ or $568 \mathrm{~nm}$ was recorded through a $515-540 \mathrm{~nm}$ band pass filter or $540-610 \mathrm{~nm}$ band pass filter, respectively. Prints from digital images were created using a Phaser IISDX dye sublimation printer (Tektronix Inc., Wilsonville, OR).

Analysis and processing of time-lapsed images were performed using the NIH-Image software package. Images were $(3 \times 3$ pixel $)$ median filtered to eliminate pixel noise from on-chip integration and quantitated with no further image processing. A background value for each image was calculated by getting the mean fluorescence of a $100 \times 100$ pixel area within the image background. Photo bleaching was calculated by taking a 1 second ( 30 frame) camera exposure of a continuously illuminated, FL-DHP labeled cell every 5 seconds for 4 minutes. Final mean fluorescence for each cell in each frame of the time lapsed series was computed by calculating the mean fluorescence of each cell and correcting for background and photobleaching. The plotted raw mean fluorescence deviated very little from corrected mean fluorescence. Local fluorescence change was calculated by selecting a small region of interest over part of the cell, measuring mean fluorescence, and correcting for frame background and photobleaching as above.

Calcium imaging was performed by confocal imaging of zygotes microinjected with either calcium green dextran $(10,000 \mathrm{Da})$ or calcium green/Texas red dextran (70,000 Da) (Molecular Probes, Eugene, OR). Microinjection was performed in ASW made hypertonic with $0.5 \mathrm{M}$ mannitol at $4{ }^{\circ} \mathrm{C}$. Cells were mounted in glass wedges and pressure microinjected with either reagent at $5 \mu \mathrm{M}$ calcium green concentration. Colocalization of calcium green dextran and the red FL-DHP ((-)-ST-BODIPY-DHP; Molecular Probes, Eugene, OR) was performed using the confocal microscope parameters listed above where cells were imaged for calcium green prior to addition of the red FL-DHP. Photomultiplier gain and offset were set such that calcium green injected zygotes did not yield any fluorescence signal when excited at $568 \mathrm{~nm}$ prior to the addition of red FL-DHP. Ratio-images were produced by confocal imaging of zygotes injected with the double labeled dextran. Autofluorescence of plastids when imaging the Texas red fluorochrome precluded accurate quantitative ratio imaging. Unprocessed images of calcium green and Texas red taken at the rhizoid tip, where autofluorescence is less prominent, were divided using the NIH-Image software package.

\section{RESULTS}

\section{FL-DHP binds a receptor localized at the plasma membrane and cell cortex}

Dihydropyridines bind to and often modulate the activity of voltage regulated L-type calcium channels (see Knaus et al., 1992). When we incubate newly fertilized Fucus zygotes with the FL-DHP $(5 \mu \mathrm{M})$ and observe their development during the first 22 hour cell cycle, we find that zygotes develop synchronously, exhibit no gross change in polar growth habit and photopolarize regardless of FL-DHP concentration (1.6 $\mu \mathrm{M}-100$ $\mu \mathrm{M})$, time of application (1-20 hours AF), or duration of application (1-20 hours). Control treatments of zygotes with solvent (ethanol) alone are indistinguishable from cells treated with only FL-DHP (data not shown).

When observed by conventional fluorescence microscopy, FL-DHP binds uniformly to newly fertilized zygotes (Fig. 1A) 

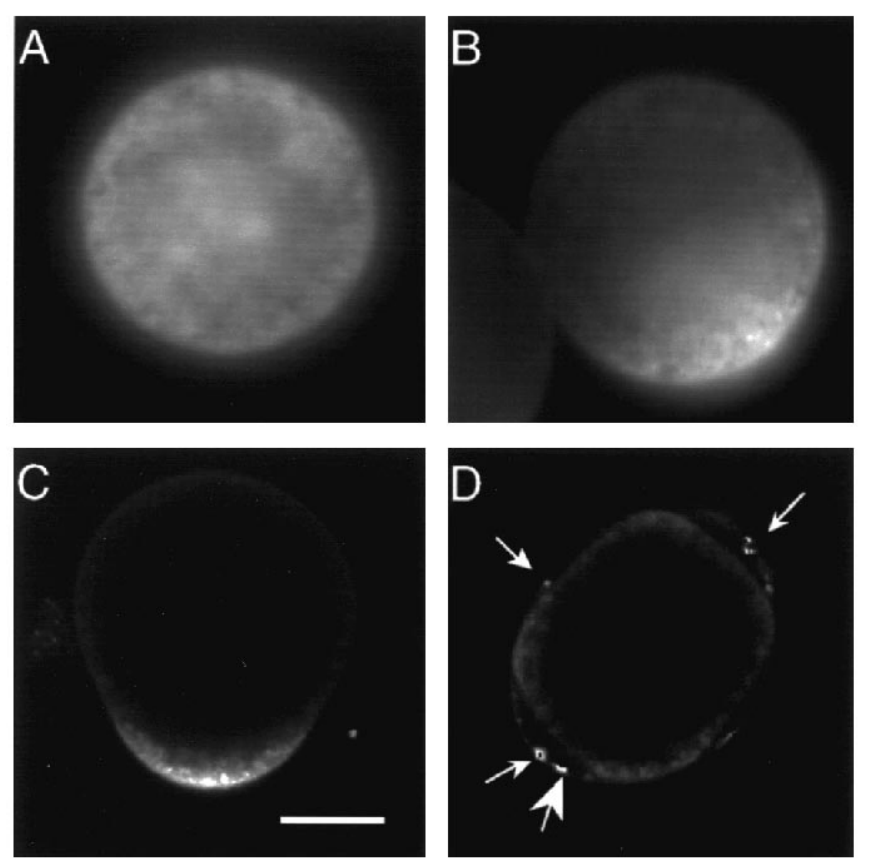

Fig. 1. Observation of FL-DHP labeled Fucus zygotes during the first 22 hour cell cycle. (A) Newly fertilized Fucus zygotes are uniformly labeled with FL-DHP. (B) Localization of FL-DHP in a morphologically symmetrical zygote at 8 hours after fertilization. (C) FL-DHP labeling of the emerging rhizoid tip in a zygote 13 hours after fertilization as visualized by confocal laser scanning microscopy. (D) Plasmolysis of the FL-DHP labeled Fucus protoplast from the cell wall. The FL-DHP brightly labels small patches of plasma membrane (arrows) that tear away with the cell wall during plasmolysis. Bar, $25 \mu \mathrm{m}$.

and asymmetrically to older zygotes (Fig. 1B) within 1 minute of application. Using laser scanning confocal microscopy to capture thin optical sections of zygotes, we observe very little initial penetrance of FL-DHP into the cell, with the primary concentration of label appearing at the cell cortex and plasma membrane (Fig. 1C). Kropf and Quatrano (1987) had shown that small patches of plasma membrane tear away with the cell wall when Fucus protoplasts are plasmolyzed in $1 \mathrm{M}$ sucrose/ASW. Under these conditions, FL-DHP remains localized to these plasma membrane patches and not to the cell wall, indicating a close association of the FL-DHP with the plasma membrane (Fig. 1D). After longer incubations, FLDHP accumulates in the perinuclear region but no other morphologically distinct localization (i.e. endoplasmic reticulum, plastids, mitochondria, Golgi) is detected. In zygotes with elongating rhizoids, a greater percentage of the FL-DHP is localized in the endomembrane system at the site of polar growth, but even at this site, labeling does not extend into the cell more than $7 \mu \mathrm{m}$ (Fig. 1C).

In order to determine the binding specificity of FL-DHP, zygotes have been preincubated (30 minutes) with Bay-K 8644, a dihydropyridine compound shown to block FL-DHP binding in animal cells (Leclerc et al., 1995). When preincubated with $100 \mu \mathrm{M}$ Bay-K 8644, application of FL-DHP does not label zygotes, rhizoid tips or the plasma membrane patches in plasmolyzed cells. The effects of Bay-K 8644 preincubation are reversible. However, longer incubations with Bay-K8644

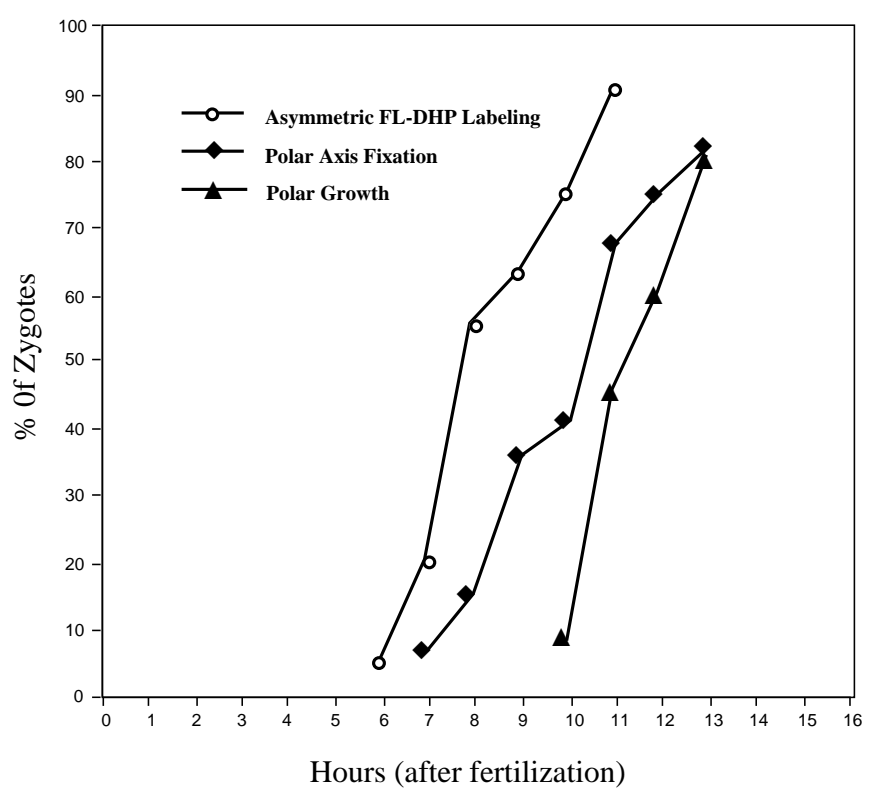

Fig. 2. The FL-DHP becomes asymmetrically distributed prior to polar axis fixation. The percentage of asymmetrically labeled zygotes in a population of cells was used to assess the relative timing of FLDHP polarization. Commitment to a polar axis and polar growth were scored as developmental markers. FL-DHP, $n=200 /$ time point; PAF, $n=400 /$ time point; polar growth, $n=200 /$ time point.

( 3 to 20 hours AF) result in the inhibition of rhizoid growth in $95 \% \pm 5 \%$ of the zygotes.

\section{Polar localization of FL-DHP receptors precedes axis fixation and is reversible}

The early symmetrical labeling of Fucus zygotes with FL-DHP changes to a highly asymmetrical localization of receptors that predicts the site of polar outgrowth (Fig. 1B). The site of FLDHP receptor localization is determined by unilateral light, and, when placed in the dark, by the position of other nearby Fucus zygotes (i.e. the positive group effect; see Kropf, 1992). Zygotes in a population start to exhibit asymmetrical fluorescent labeling at 6 hours after fertilization (AF), with $50 \%$ of the cells showing asymmetry in FL-DHP receptors by 8 hours AF (Fig. 2). Zygotes in the same population first exhibit PAF at 8 hours AF, with half of the cells committing to a polar axis by 10 hours AF (Fig. 2). Polar growth of the rhizoid follows axis fixation by approximately 2 hours (Fig. 2). Interestingly, regardless of signal intensity (0 lux to 3,000 lux) or when the light treatment is given prior to 6 hours $\mathrm{AF}$, polar localization of the FL-DHP does not begin before 6 hours AF.

Since the distribution of FL-DHP becomes asymmetrically localized before polar axis fixation, the position of the FL-DHP receptors should be altered by a change in the direction of unilateral light. To determine if the asymmetric localization of dihydropyridine receptors is a marker for polar axis formation, zygotes are incubated in unilateral light until the zygotes are asymmetrically fluorescent (Fig. 3A). At various times after zygotes exhibit FL-DHP asymmetry, unilateral light is applied to the opposite side of the zygote and the FL-DHP is later imaged (Fig. 3B). We find that the position of the FL-DHP receptors is reversed if unilateral light is applied from an opposing direction before 10 to 11 hours AF. In addition, the fluorescence becomes 
more intense on the side opposite from the unilateral light within 45 minutes to 1 hour from the time of light reversal $(n=5)$.
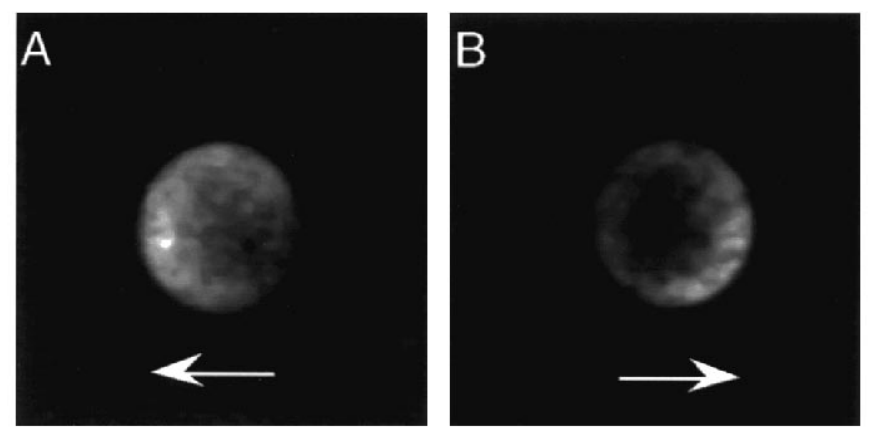

Fig. 3. Asymmetrical distribution of FL-DHP can be redistributed prior to polar axis fixation by reversing the position of unilateral white light on the zygote. (A) FL-DHP labeled zygote at 7 hours after fertilization in unilateral white light. (B) The localization of FLDHP on the same zygote has relocated to the opposite side of the zygote 60 minutes after reversing the position of unilateral light. Attempts to repolarize DM-B in zygotes $>11$ hours after fertilization were unsuccessful.

\section{Existing FL-DHP receptors are redistributed during polarization}

Using digital time-lapse fluorescence microscopy, we have followed the development of FL-DHP receptor asymmetry in zygotes by taking a fluorescence image at 7.5 minute intervals and importing the image directly into the computer for analysis (see Materials and Methods) (Fig. 4A). We find that when FLDHP fluorescence is measured for the entire Fucus zygote at each time point, the total fluorescence is constant for the duration of the experiment (Fig. 4B). Furthermore, when a region of the cell representing the site of future rhizoid growth ( $\sim 6 \%$ of the cell image) is measured, the local mean fluorescence increases substantially (Fig. 4B) relative to the mean fluorescence for the entire cell. Photobleaching, as analyzed by taking images at several time points under continuous illumination, is small for the aggregate time of illumination in this time-lapse series.

\section{Cytochalasin B reversibly blocks FL-DHP Iocalization}

It has been demonstrated previously that F-actin is required for polar axis formation (Quatrano, 1973). When $50 \mu \mathrm{M}$ cyto-
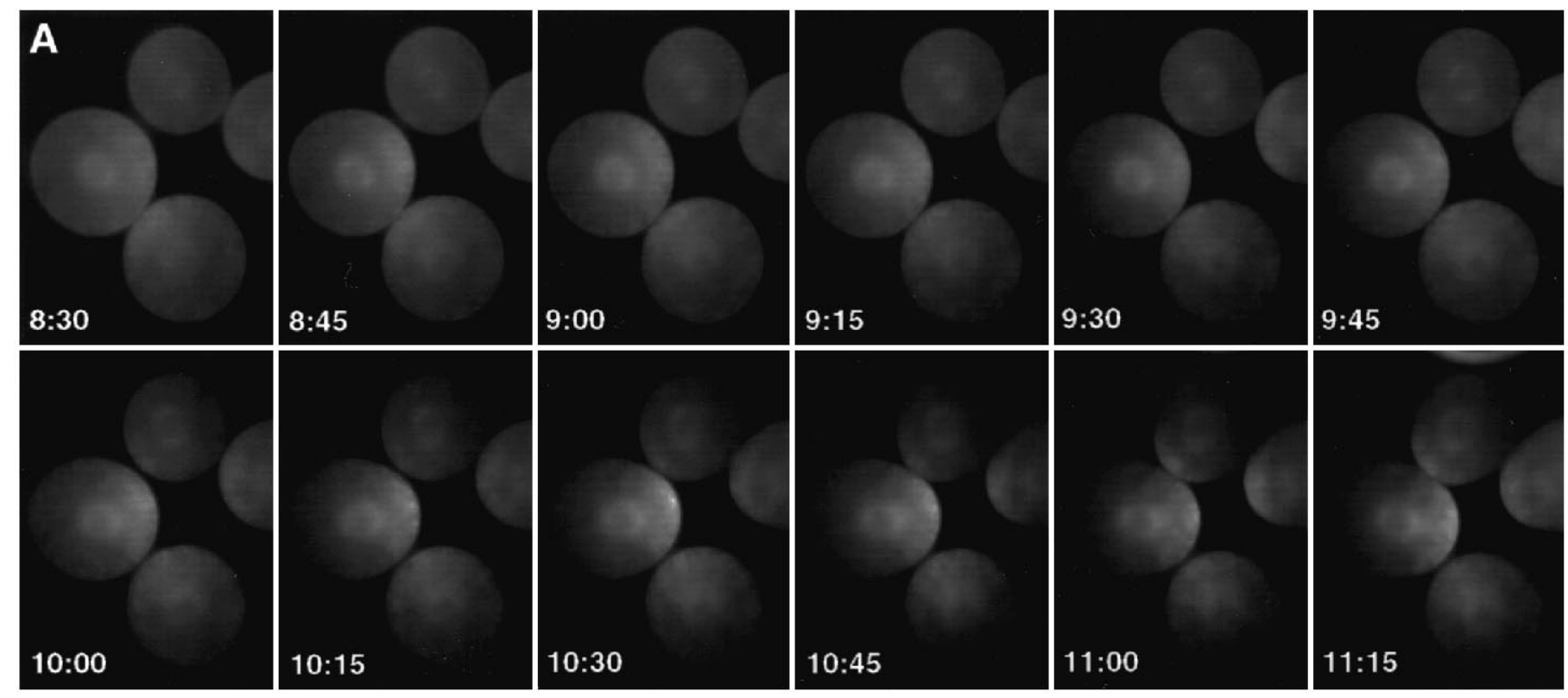

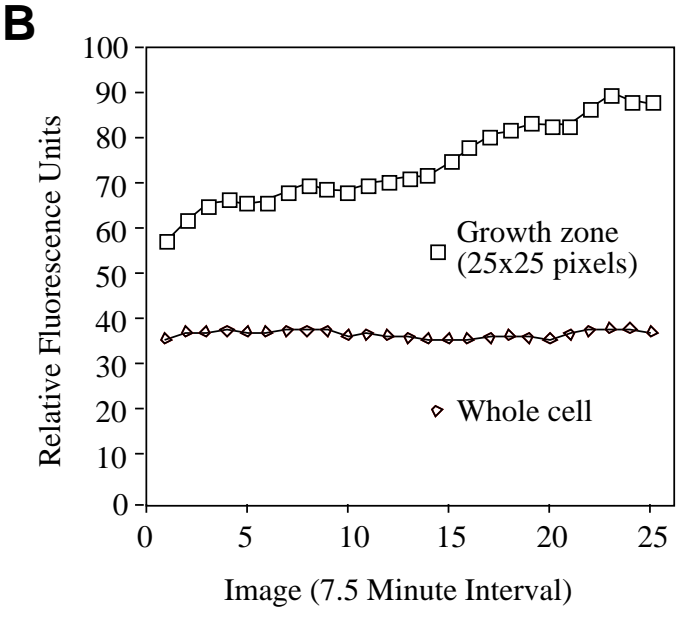

Fig. 4. Time-lapse fluorescence microscopy of polarizing FL-DHP. (A) A series of 12 images taken at 15 minute time intervals of living Fucus zygotes labeled with FL-DHP during polarity establishment. (B) The mean fluorescence for each zygote every 7.5 minutes is plotted (average of three cells) over a time course of 3 hours indicating that total fluorescence does not change during polarization. A region of interest ( $\sim 6 \%$ of total cell image area) was selected at the brighter side of each zygote and the mean fluorescence was plotted (average of three cells) over time. 
chalasin B (CB) is added to zygotes from 4-13 hours AF (Fig. $5 \mathrm{~A}$ ) (untreated cells already possess a fixed polar axis at 13 hours AF), FL-DHP receptors are distributed symmetrically (Fig. 5C). When CB is replaced with fresh ASW, the FL-DHP becomes asymmetrically localized within 45 minutes of $\mathrm{CB}$ removal (Fig. 5D). Untreated cells (Fig. 5B) and cells treated with $50 \mu \mathrm{M}$ colchicine or $0.5 \%$ DMSO (data not shown) all show a normal pattern of polar FL-DHP localization.

\section{FL-DHP receptors colocalize with increased calcium levels in the cortical cytoplasm}

A calcium ion gradient had been previously measured in Fucus rhizoid cells using the calcium reporter dye, calcium green dextran (Berger and Brownlee, 1993). To determine if the distribution pattern of FL-DHP receptors is consistent with FLDHP binding to calcium channels, FL-DHP is colocalized with intracellular calcium gradients. Zygotes pressure injected with $5 \mu \mathrm{M}$ calcium green dextran are labeled with a contrasting red FL-DHP and visualized by confocal microscopy using two different excitation wavelengths and mutually exclusive emission filters. Imaging of calcium green fluorescence is executed prior to application of the red FL-DHP to insure that the calcium green fluorescence is not a result of red FL-DHP binding. Calcium green fluorescence is consistently higher at the rhizoid tips (Fig. 6A), as previously reported (Berger and Brownlee, 1993). Furthermore, we routinely detect elevated fluorescence intensity at the site on the cortex $(n=15)$ that predicts the location of polar growth (Fig. 6C). And, when we observe an increase in calcium green fluorescence, a superimposable increase in red FL-DHP fluorescence is always observed (Fig. 6D).

In order to rule out the possibility that the increased calcium
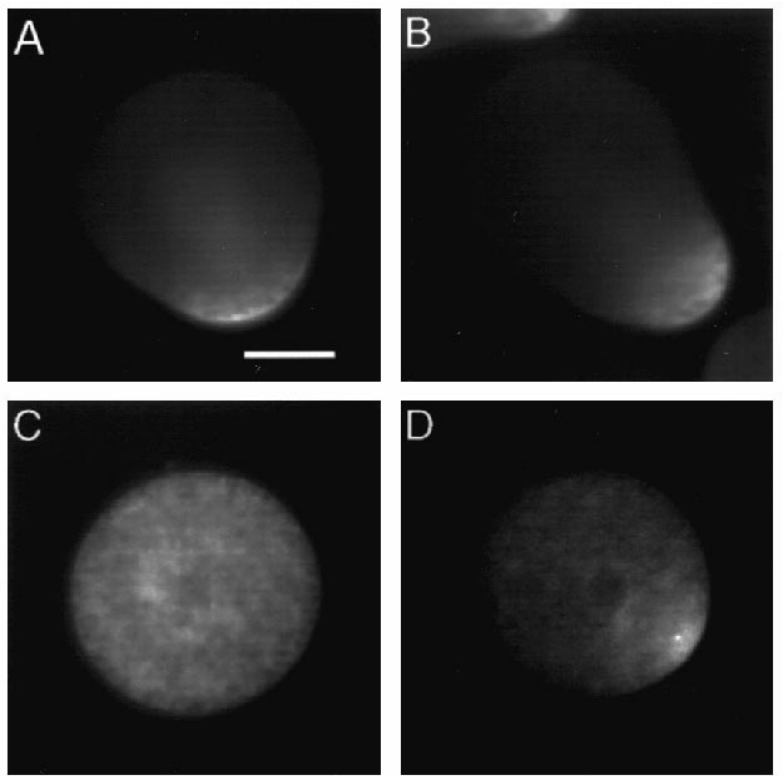

Fig. 5. Cytochalasin B reversibly blocks FL-DHP polarization. FLDHP labeling of untreated zygotes at 13 hours (A) and at 14 hours (B) after fertilization. (C) Zygote treated with $20 \mu \mathrm{g} / \mathrm{ml} \mathrm{CB}$ beginning at 7 hours after fertilization has uniform FL-DHP labeling and no polar growth at 13 hours after fertilization. (D) When the CB is replaced with fresh ASW at 13 hours after fertilization, FL-DHP begins to asymmetrically localize within 60 minutes. Bar, $25 \mu \mathrm{m}$. green dextran signal is the result of dextran actively transported to the site of FL-DHP fluorescence, a double labeled calcium green/Texas red dextran is injected and imaged in a confocal microscope. Accurate quantitative ratio imaging is not possible due to the autofluorescence of plastids present when imaging the Texas red fluorochrome. However, confocal ratio images of the rhizoid tip, where autofluorescence is less, confirm that the elevated fluorescence observed using calcium green is not the property of an increase in dye concentration (Fig. 6E,F,G). As previously reported (Berger and Brownlee, 1993), the calcium green dextran retards the development of most Fucus zygotes relative to uninjected cells, thereby preventing an
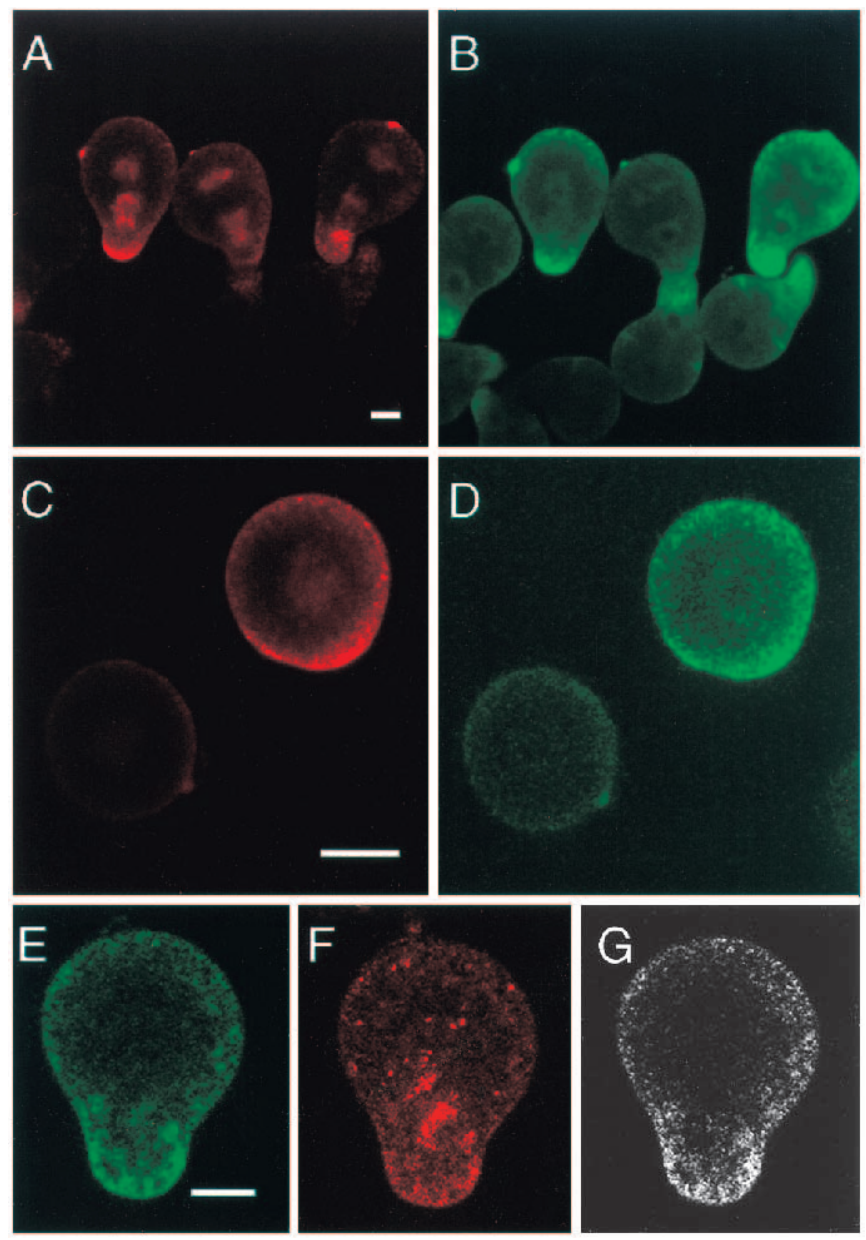

Fig. 6. The FL-DHP is associated with a cortical calcium gradient. Zygotes injected with calcium green dextran $(A, C)$ were also labeled with FL-DHP (ST-BODIPY-DHP) (B,D). Note that only the top three cells labeled with FL-DHP in B were microinjected with calcium green dextran and appear in A. Calcium green dextran fluorescence is highest at the tips of the Fucus rhizoid (A) and correlates with the localization of FL-DHP labeling (B). The presence of high calcium green fluorescence occurs just prior to polar growth (C). The less prominantly fluorescent cell in C and D was injected with calcium green dextran and does not appear to have a gradient of either calcium green fluorescence or FL-DHP fluorescence. Fucus zygote injected with a double labeled calcium green/Texas red dextran was imaged first for calcium green $(\mathrm{E})$ and then Texas red $(F)$ for calculation of the ratio image $(\mathrm{G})$. Calcium green fluorescence appears brighter at the rhizoid tip in a ratioimaged cell (G). Bar, $25 \mu \mathrm{m}$. 
accurate assessment of the developmental state of the cell. For this reason, it is not possible to resolve exactly when the increase in calcium green dextran fluorescence occcurs relative to axis formation or axis fixation. However, a localized increase in fluorescence at the cell cortex prior to polar growth is consistantly observed $(n=15)$ for calcium green dextran injected cells. Hence, it appears that the localized FL-DHP receptors may correspond to ion channels concentrated at the site of polar growth responsible for the increase in cytoplasmic calcium at that cytoplasmic site.

\section{DISCUSSION}

\section{FL-DHP binds to a dihydropyridine receptor localized at the plasma membrane}

FL-DHP labels live Fucus zygotes predominantly at the cell periphery. We interpret this staining pattern as including the plasma membrane and vesicles very closely underlying the plasma membrane. Further supporting evidence includes the preferential labeling of cell wall associated plasma membrane patches formed when Fucus protoplasts are plasmolysed away from the cell wall (Kropf and Quatrano, 1987). Eventual localization of FL-DHP to the perinuclear region could not be readily interpreted as we have found that many hydrophilic fluorescent molecules (e.g. lucifer yellow, fluorescein) introduced into the ASW medium are eventually localized to a region surrounding the nucleus (S. L. Shaw, unpublished observation).

Preincubation of Fucus zygotes with Bay-K 8644, an unlabeled dihydropyridine compound, specifically inhibited the binding of FL-DHP at the cell cortex, indicating that FLDHP was binding to dihydropyridine receptors. The ability of FL-DHP to label zygotes could be restored to Bay-K 8644 treated zygotes by extensive washing in fresh ASW, indicating that the dihydropyridine receptors were still present after Bay-K 8644 treatment. Unconjugated fluorochrome did not show any of the localization properties of the FL-DHP, and staining of Fucus zygotes with fluorochrome was not inhibited by preincubation with Bay-K 8644. Furthermore, addition of Bay-K 8644 inhibited polar growth of the rhizoid, consistent with results from the addition of other putative calcium channel binding drugs to Fucus zygotes (Kropf and Quatrano, 1987). Bay-K 8644 has previously been used to block FL-DHP binding in animal cells (Leclerc et al., 1995) and has been used in several calcium related plant studies (Chen and Wolniak, 1987; Schumaker and Gizinski 1993). From these data, we conclude that the FL-DHP binds to a dihydropyridine receptor on Fucus zygotes localized primarily at the plasma membrane.

\section{FL-DHP receptors are physically redistributed during polar axis formation in an actin dependent process}

Observations of FL-DHP fluorescence on living Fucus cells using time-lapse video microscopy indicates that the FL-DHP receptor is physically redistributed during polar axis formation. Quantitative analysis of the images taken during FL-DHP polarization shows that the total amount of FL-DHP fluorescence does not change over the course of polarization. We interpret this to mean that the number of FL-DHP receptors resident on the Fucus cell remained constant during the polarization process. We further showed that there is a substantial increase in fluorescence on one side of the Fucus cell. Since the total amount of FL-DHP bound to the zygote does not change while FL-DHP accumulates at the site targeted for polar growth, the simplest interpretation of these analyses is that the FL-DHP receptor is being physically redistributed from a symmetrical to an asymmetrical arrangement on the zygote surface during polarization. Attempts at following a photobleached spot on the FL-DHP labeled cells to further address the idea of cortical translocation have not been successful due to cells being damaged by the high levels of irradiance required to bleach the BODIPY fluorophore (S. L. Shaw, unpublished observation).

If the asymmetrical FL-DHP distribution is a result of localized uptake of FL-DHP from the medium, or if the increase in fluorescence is the property of a change in fluorochrome quantum efficiency due to some local environmental effect (e.g. pH, pCa, lipid composition, membrane potential), then the total measured fluorescence would have been expected to increase over time as the cell became polarized. Photobleaching or photoinactivation of the FL-DHP molecule by either unilateral white light or blue light used for epifluorescence microscopy has been ruled out as being causative to the change in FL-DHP fluorescence distribution as asymmetrical FL-DHP localization occurs normally in omnidirectional light and in the dark. We cannot entirely rule out the possibility that FL-DHP polarization is occurring by a transcytotic or other secretion based mechanism. However, the majority of FL-DHP labeling was at the cell cortex and plasma membrane and there was no obvious polarized fluorescent vesicle traffic observable within the cell as judged by conventional or confocal microscopy of live cells. Further, the redistribution of FL-DHP fluorescence occurred even when the majority of free FL-DHP was removed from the medium using activated charcoal.

\section{FL-DHP receptors are associated with increased cytoplasmic calcium ion levels}

The evidence presented in this work indicates that the FL-DHP is associated with elevated calcium concentrations at the cell cortex in Fucus. FL-DHP has been shown to label dihydropyridine receptors and has been used to purify calcium channels in animal cells (Leclerc et al., 1995; Berger et al., 1994; Knaus et al., 1992). The FL-DHP labels predominantly at the cortex of Fucus zygotes, including the plasma membrane and plasma membrane patches attached to the cell wall which have previously been shown to contain calcium (Kropf and Quatrano, 1987). FL-DHP is also concentrated at the rhizoid tip, the site of polar growth. Studies using electrophysiological and ratiometric dye methods to measure calcium have consistently demonstrated the presence of a high calcium gradient in the tips of Fucus rhizoids (Berger and Brownlee, 1993). The striking similarity of the FL-DHP labeling to published ratiometric images of calcium gradients prompted us to attempt the colocalization of the FL-DHP and a calcium sensitive fluorescent dye in living Fucus cells. Due to the limited fluorescent spectrum available in plant systems, created by the autofluorescence of chloroplasts and other plastids, we are unable to use a ratiometric calcium indicator simultaneously with the FLDHP. Using the same calcium sensitive dye previously selected for use in Fucus, we observe that all local increases in calcium green fluorescence at the cell cortex were superim- 
posable upon the site of increased FL-DHP fluorescence. Ratio-imaging of the calcium sensitive dye with a calcium insensitive dye coupled to the same carrier molecule indicated that the increased fluorescence was a property of a change in calcium green fluorescence and not the property of nonuniform dye distribution. These data, taken together with the specific inhibition of FL-DHP binding by the unlabeled dihydropyridine Bay-K 8644, suggest strongly that the Fucus FLDHP receptor is an ion channel, possibly a calcium channel, at the Fucus plasma membrane.

Using an ion sensitive vibrating probe, Nuccitelli (1978) discovered that a localized ion influx in Pelvetia zygotes, partially composed of calcium ions, predicts the site of polar growth. Nuccitelli (1978) also discovered that the point of ion influx could be relocated on the cell cortex prior to the onset of polar growth, by changing the position of incident unilateral white light. Brawley and Robinson (1985) later demonstrated, also with a vibrating probe, that cytochalasin prevents the localized ion influx. The characteristics of asymmetric FL-DHP localization in Fucus cells are similar to properties of the externally measured ion influx observed in Pelvetia; the FL-DHP is localized to the cell cortex and plasma membrane, becomes asymmetrically localized in response to unilateral white light before polar growth, predicts the site of polar growth, and does not asymmetrically localize in the presence of CB. Perhaps the most striking similarity is that both the ion influx observed by Nuccetelli (1978) and FL-DHP localization are relocated on the cell cortex prior to the time of polar axis fixation by changing the position of incident unilateral white light. We conclude that the FL-DHP is a visible marker for formation of a labile polar axis, and suggest that it may mark the ion channels which give rise to the asymmetrically localized ion influx.

\section{Significance of FL-DHP as a marker for polar axis formation}

The general aim of our studies is to elucidate the mechanisms by which the symmetric Fucus zygote becomes asymmetric during the first 22 hour cell cycle. Many very different external gradients, such as light, heat, ions, and the position of other Fucus zygotes, can be used to orient this developmental axis. Hence, several different signaling pathways are present in zygotes, to perceive and transmit these different external cues or gradients to some common intermediate resulting in polar axis formation. We believe that the asymmetric distribution of FL-DHP receptors marks a late stage in polar axis formation and is the end result of different signaling pathways.

Asymmetric distribution of FL-DHP receptors is clearly part of axis formation and not axis fixation, since the initial asymmetrical FL-DHP distribution is reversible and occurs before axis fixation. Furthermore, both unilateral light and the presence of a nearby zygote (i.e. the positive group effect) result in the localization of FL-DHP receptors, indicating that perception of two different external cues by zygotes leads to the same asymmetry. Interestingly, although FL-DHP receptors do not become asymmetrically distributed prior to 6 hours AF, regardless of the amount of incident light flux (from darkness to 3,000 lux), cells are able to interpret a light gradient at 3 hours AF. This result clearly indicates that polarization of the FL-DHP receptors is not the first event in polar axis formation or signal perception. Thus, our data suggests that neither the initial perception of light nor the 'memory' of the light direction is reflected by the localization of the FL-DHP. Although we have shown that localization of FL-DHP receptors requires microfilaments, $\mathrm{CB}$ treatment does not affect these earlier events in polar axis formation, i.e. the perception of light or memory of its direction (Quatrano, 1973). Our data suggest, then, that the actin-dependent localization of the FLDHP receptor is the downstream expression of an asymmetry signaled by any one of several different vectoral stimuli. Furthermore, the timing of FL-DHP localization appears to be under the control of a developmental clock and not the orienting signal. We will now be able to use FL-DHP localization as a visual assay to identify earlier intermediates in the process of polar axis formation (i.e. those associated with light perception and memory of light direction) when perturbed with various inhibitors, etc.

We conclude in this study that the FL-DHP receptor is likely to be an ion channel on the basis of the effects of dihydropyridine molecules in animal and plant cells and the exact colocalization of the FL-DHP receptor with a calcium ion gradient in the cortical cytoplasm. We also demonstrate, by time-lapse video microscopy, that the likely mechanism for localizing FLDHP receptors to a specific site at the cell cortex is a physical translocation in the plasma membrane. The results presented in this study, and the report of a localized ion influx (Nuccitelli, 1978; Brawley and Robinson, 1985), suggest that an intact Factin cytoskeleton is required for the proper localization or activation of ion channels in the plasma membrane. Our data extend the previous observations by providing visual evidence that ion channels (FL-DHP receptors) are physically localized and require the actin cytoskeleton for their translocation to the site of polar growth. This process may be analogous to the capping mechanism by which some animal cell types translocate plasma membrane bound receptors to one side of the cell in an actin/myosin dependent fashion (Albertini et al., 1991; Bourguignon et al., 1993).

We propose from these studies that the ion channels which are translocated within the plasma membrane to a specific location on the cell surface give rise to a cytoplasmic ion gradient. The colocalization of the surface FL-DHP receptor with a subtending concentration of calcium ions supports the idea that the ion channels we are labeling with the FL-DHP are calcium channels. Treatments with calcium channel antagonists (Kropf and Quatrano, 1987) and microinjection of calcium buffers (Speksnijder et al., 1989) have demonstrated a requirement for calcium ions for polar growth but no evidence has been presented previously for a direct role(s) for calcium ions in the establishment of polarity. We predict, from this study, that calcium ion gradients are not required for the early selection or formation of the polar axis because the calcium channels responsible for establishing the gradient are not localized until the later stage of axis formation. One possible role for the observed calcium ion gradient, which we propose to be one of the latest events of polar axis formation, is to define a target site for localized secretion. Treatment with $\mathrm{CB}$, calcium channel antagonists, and injected calcium buffers all prevent polarized secretion. Further, Nuccetelli (1978) demonstrated localized jelly secretion at the point of ion influx in Pelvetia zygotes. Observations by Kropf et al. (1988), that an intact cell wall is required to fix a polar axis, and by Berger et al. (1994), that the cell wall contains molecules with 
signaling capacities, suggest that a calcium-induced polarized secretion of molecules into the cell wall may play a key role in the subsequent events of polar axis fixation and cell fate determination.

The authors thank Drs J. Fowler, L. Smith, and C. Taylor for comments on the original manuscript. This work was supported by grants from NSF \#MCB9318757 and ONR \#N00014-93-1-0888 to R.S.Q.

\section{REFERENCES}

Albertini, D., Berlin, R. and Oliver, J. (1991). The mechanism of Con A cap formation in leukocytes. J. Cell Sci. 26, 57-75.

Berger, F. and Brownlee, C. (1993). Ratio confocal imaging of free cytoplasmic calcium gradients in polarising and polarised Fucus zygotes. Zygote 1, 9-15.

Berger, W., Prinz, H., Striessnig, J., Kang, H. C., Haugland, R. and Glossmann, H. (1994). Complex molecular mechanism for dihydropyridine binding to L-type $\mathrm{Ca}\left({ }^{2+}\right)$-channels as revealed by fluorescence resonance energy transfer. Biochemistry 33, 11875-83.

Berger, F., Taylor, A. and Brownlee, C. (1994). Cell fate determination by the cell wall in early Fucus development. Science 263, 1421-1423.

Bourguignon, L. Y., Lokeshwar, V. B., Chen, X. and Kerrick, W. G. (1993). Hyaluronic acid-induced lymphocyte signal transduction and HA receptor (GP85/CD44)-cytoskeleton interaction. J. Immunol. 151, 6634-44.

Brawley, S. H. and Robinson, K. R. (1985). Cytochalasin treatment disrupts the endogenous currents associated with cell polarization in fucoid zygotes: studies of the role of F-actin in embryogenesis. J. Cell Biol. 100, 1173-84.

Chen, T. L. and Wolniak, S. M. (1987). Mitotic progression in stamen hair cells of Tradescantia is accelerated by treatment with ruthenium red and Bay-K 8644. Eur. J. Cell Biol. 45, 16-22.

Fowler, J. and Quatrano, R. S. (1995). Cell polarity, asymmetric division, and cell fate determination in brown algal zygotes. In Seminars in Developmental Biology: Simple Systems for the Analysis of Important Developmental Problems, vol. 6 (ed. D. Kirk), pp. 347-358. Academic Press, Cambridge, UK.

Goodner, B. and Quatrano, R. S. (1993). Fucus embryogenensis: A model to study the establishment of polarity. The Plant Cell 5, 1471-1481.

Hülskamp, M., Misra, S. and Jurgens, G. (1994). Genetic dissection of trichome cell development in Arabidopsis. Cell 76, 555-66.

Jaffe, L. A. (1966). Electrical currents through the developing Fucus egg. Proc. Nat. Acad. Sci. USA 56, 1102-1109.

Jürgens, G., Mayer, U., Ruiz, R. A. T., Berleth, T. and Miséra, S. (1991). Genetic analysis of pattern formation in the Arabidopsis embryo. Development (Suppl. ) 1, 27-38.

Jürgens, G. (1995). Axis formation in plant embryogenesis: cues and clues. Cell 81, 467-470.

Knaus, H. G., Moshammer, T., Friedrich, K., Kang, H. C., Haugland, R. P. and Glossman, H. (1992). In vivo labeling of L-type $\mathrm{Ca}^{2+}$ channels by fluorescent dihydropyridines: evidence for a functional, extracellular heparin-binding site. Proc. Nat. Acad. Sci. USA 89, 3586-90.

Kropf, D. L. and Quatrano, R. S. (1987). Localization of membraneassociated calcium during development of fucoid algae using chlorotetracycline. Planta 171, 158-170.

Kropf, D. L., Kloareg, B. and Quatrano, R. S. (1988). Cell wall is required for fixation of the embryonic axis in Fucus zygotes. Science 239, 187-90.

Kropf, D. L., Berge, S. K. and Quatrano, R. S. (1989). Actin localization during Fucus embryogenesis. The Plant Cell 1, 191-200.

Kropf, D. L. (1992). Establishment and expression of cellular polarity in fucoid zygotes. Microbiol. Rev. 56, 316-339.

Kropf, D. L. (1994). Cytoskeletal control of cell polarity in a plant zygote. Dev. Biol. 165, 361-71.

Leclerc, C., Duprat, A.-M. and Moreau, M. (1995). In vivo labeling of L-type calcium channels by fluorescent dihydropyridine: correlation between ontogenesis of the channels and the acquisition of neural competence in ectoderm cells from Pleurodeles wactl embryos. Cell Calcium 17, 216-224.

McCormick, S. (1993). Male gametophyte development. The Plant Cell 5, 1265-1275.

Nuccitelli, R. (1978). Ooplasmic segregation and secretion in Pelvetia egg is accompanied by the membrane-generated electrical current. Dev. Biol. 62, 13-33.

Quatrano, R. S. (1973). Separation of processes associated with the differentiation of two-celled Fucus embryos. Dev. Biol. 30, 209-213.

Quatrano, R. S. (1978). Development of cell polarity. Annu. Rev. Plant Physiol. 29, 487-510.

Quatrano, R. S., Brawley, S. H. and Hogsett, W.E. (1979). The control of the polar deposition of sulfated polysaccharide in Fucus zygotes. In Determinants of Spatial Organization (ed. S. Subtelny and I. R. Konigsbergs), pp. 77-96. New York: Academic Press.

Quatrano, R. S. (1990). Polar axis fixation and cytoplasmic localization in Fucus. In Genetics of Pattern Formation and Growth Control. pp. 31-46. Wiley-Liss, Inc.

Reiser, L. and Fischer, R. L. (1993). The ovule and embryo sac. The Plant Cell 5, 1291-1301.

Robinson, K. R. and Jaffe, L. F. (1975). Polarizing fucoid eggs drive a calcium current through themselves. Science 187, 70-72.

Schumaker, K. S. and Gizinski, M. J. (1993). Cytokinin stimulates dihydropyridine-sensitive calcium uptake in moss protoplasts. Proc. Nat. Acad. Sci. USA 90, 10937-41.

Shaw, S. L., Salmon, E. D. and Quatrano, R. S. (1995). Digital photography for the light microscope: results with a gated video rate CCD camera and NIH-image. Biotechniques (in press)

Speksnijder, J. E., Miller, A. L., Weisenseel, M. H., Chen, T. H. and Jaffe, L. F. (1989). Calcium buffer injections block fucoid egg development by facilitating calcium diffusion. Proc. Nat. Acad. Sci. USA 86, 6607-11.

Wick, S. M. (1991). Spatial aspects of cytokinesis in plant cells. Curr. Opin. Cell Biol. 3, 253-260.

(Received 12 May 1995 - Accepted 31 October 1995) 